\title{
Propriedades físicas do solo e crescimento de eucalipto implantado em diferentes métodos de preparo do solo
}

\author{
Soil physical properties and eucalyptus \\ growth planted after different tillage methods
}

\author{
Samara Pozzan da Rocha1, Juliana Prevedello², Dalvan José Reinert ${ }^{3}$, \\ Frederico Dimas Fleig ${ }^{4}$, Eduardo Saldanha Vogelmann², \\ Júlio Cesar Wincher Soares ${ }^{1}$ e Breno Bevilaqua Heinz ${ }^{5}$
}

\section{Resumo}

O objetivo do estudo foi verificar alterações nas propriedades físicas de um Argissolo Vermelho-Amarelo distrófico arênico e no crescimento de Eucalyptus grandis, implantado em diferentes métodos de preparo do solo, após 6 anos. O experimento foi realizado na Fundação Estadual de Pesquisa Agropecuária, em Santa Maria, Rio Grande do Sul. O delineamento experimental utilizado foi blocos ao acaso com três repetições e quatro tratamentos: coveamento manual, escarificado, escarificado mais grade niveladora e enxada rotativa. Antes e 72 meses após o preparo do solo foram coletadas amostras de solo indeformadas nas camadas de $0-0,1 ; 0,1-0,2 ; 0,2-0,3$ e $0,3-0,4$ m, para a determinação da macroporosidade, microporosidade, porosidade total e densidade do solo. A distribuição do tamanho dos agregados estáveis em água e o teor de matéria orgânica do solo foram determinados na camada de 0-0,05 $\mathrm{m}$. O crescimento do eucalipto foi avaliado aos 74 meses de idade das árvores pela medida da altura total, do diâmetro e da sobrevivência. Seis anos após o preparo do solo não ocorreu diferença significativa entre tratamentos para as propriedades físicas do solo nem para o crescimento do eucalipto. Todavia, houve efeito negativo do coveamento manual sobre a sobrevivência das árvores. Nesse mesmo período de tempo, foi observada melhoria das propriedades físicas do solo devido ao aumento da porosidade e redução da densidade do solo na camada superficial.

Palavras-chave: qualidade física do solo; Eucalyptus grandis; manejo do solo; estrutura do solo.

\begin{abstract}
The objective of this study was to determine changes in the physical properties of an Alfisol and in the growth of Eucalyptus grandis, established under different soil tillage methods, after 6 years. The experiment was conducted at the State Foundation for Agricultural Research in Santa Maria, Rio Grande do Sul. The experimental design used was randomized blocks with three replications and four treatments: manual hoeing, chisel plow, chisel plow plus harrowing and rotary hoe. Undisturbed soil samples were collected from depths of $0-0.10 ; 0.10-0.20 ; 0.20-0.30$ and $0.30-0.40 \mathrm{~m}$ before and seventy-two months after the soil preparation in order to determine the macro porosity, micro porosity, total porosity and bulk density. The size distribution of water stable aggregates and the organic matter content of the soil were determined in the 0-0.05 m layer. Eucalyptus growth was evaluated at seventy-four months old by measuring height, diameter and survival of trees. Six years after soil preparation there were no significant differences between treatments for the soil physical properties and eucalyptus growth. However, there was a negative effect from manual hoeing on the trees survival. In the same period an improvement of soil physical properties by reducing density and increasing porosity in the surface layer was observed, highlighting the influence of soil and root system organic matter as well as litter from trees.
\end{abstract}

Keywords: soil physical quality; Eucalyptus grandis; soil management; soil structure.

\footnotetext{
${ }_{1}^{1}$ Doutorando(a) em Engenharia Florestal. UFSM - Universidade Federal de Santa Maria - Centro de Ciências Rurais. Av. Roraima, 1000, - Camobi - 97105-900 - Santa Maria, RS. E-mail: samarapozzan@gmail.com

2Professor(a) Doutor (a). FURG - Universidade Federal do Rio Grande. Rua Marechal Floriano Peixoto, 2236 - Centro 96170-000 - São Lourenço do Sul, RS. E-mail: julianaprevedello@furg.br; eduardovogelmann@furg.br

${ }^{3}$ Professor Titular do Departamento de Solos. UFSM - Universidade Federal de Santa Maria - Centro de Ciências Rurais. Av. Roraima, 1000, Sala 3309 - Camobi - 97105-900 - Santa Maria, RS. E-mail: dalvan@ufsm.br

${ }^{4}$ Professor Adjunto - UFSM - Universidade Federal de Santa Maria - Centro de Ciências Rurais - Departamento de Ciências Florestais - Av. Roraima, 1000, Sala 5249 - Camobi - CEP: 97105-900 - Santa Maria, RS.

${ }^{5}$ Graduação em agrônomia. UFSM - Universidade Federal de Santa Maria - Centro de Ciências Rurais. Caixa Postal, 281 Av. Roraima, 1000, - Camobi - 97105-900 - Santa Maria, RS. E-mail: breno bevilaqua@ hotmail.com
} 


\section{INTRODUÇÃO}

Do ponto de vista agrícola e florestal, uma das propriedades físicas do solo mais importante é a estrutura, a qual está relacionada com outras propriedades que são fundamentais nas relações solo-planta e que determinam a qualidade do solo. A estrutura está relacionada com a densidade, a porosidade e a permeabilidade do solo, assim como com a resistência do solo à penetração das raízes (LETEY, 1985; SILVA et al., 2011).

A qualidade estrutural do solo pode ser avaliada por meio da estabilidade dos agregados, pois a estabilidade da estrutura está relacionada à resistência que os agregados apresentam à influência da água, do vento e de forças mecânicas (KIEHL, 1979). Agregados maiores e mais estáveis conferem maior resistência à erosão e maior infiltração de água. Entretanto, agregados menores e menos estáveis tendem a desaparecer e se dispersarem (BERTONI; LOMBARDI NETO, 2008), causando a redução da taxa de infiltração da água, aumento do encrostamento e do escoamento superficial, podendo afetar o crescimento das plantas (PICCOLO et al., 1997).

A densidade do solo reflete as condições estruturais do solo, visto que considera o espaço poroso, o qual é uma consequência de como estão arranjadas as partículas primárias do solo. De modo geral, quanto maior a densidade do solo mais compactado ele será, menor será o grau de estruturação, menor será a porosidade e, consequentemente, maiores serão as restrições ao crescimento radicular das plantas. A porosidade do solo é de grande importância para as plantas porque nos poros ocorrem fenômenos que fomentam as condições favoráveis ao crescimento do sistema radicular, como as reações químicas e biológicas, fluxo de fluídos e difusão de gases e íons (AMARO FILHO et al., 2008).

A densidade e a porosidade do solo dependem da agregação das partículas do solo, da composição mineralógica da fração argila, da textura, da profundidade do solo, da cobertura vegetal, do teor de matéria orgânica e do uso e manejo do solo (AMARO FILHO et al., 2008; FERREIRA, 2010). A influência da cobertura vegetal ocorre por meio das raízes e da serapilheira, a qual fornece nutrientes para a biota do solo. A interação das plantas e da biota resulta na construção da estrutura física do solo, cuja complexidade é maior quanto maior a quantidade, qualidade, diversidade e frequência do aporte da serapilheira (VEZANI; MIELNICZUK, 2009). A deposição de serapilheira varia conforme a composição de espécies, a idade das árvores e o tipo de floresta (nativa ou plantada) (NEVES et al., 2001). Braida et al. (2011) reportam que a matéria orgânica do solo, proporcionada pela serapilheira, interfere no comportamento físico do solo atuando na formação de agregados e, portanto, na distribuição do tamanho de poros, assim como na sua estabilidade.

Em relação ao efeito do manejo sobre as propriedades físicas do solo, Prevedello (2008) observou que o revolvimento do solo causou redução da densidade e aumento da porosidade total e da macroporosidade do solo. Além disso, constatou que as forças disruptivas da ação mecânica das máquinas agrícolas causaram aumento da percentagem de agregados de menor tamanho e redução da percentagem de maior tamanho. Além da ação disruptiva das máquinas, o revolvimento do solo faz com que a oxidação dos compostos orgânicos seja maior, diminuindo a ação cimentante da matéria orgânica e, consequentemente, reduzindo a agregação das partículas do solo (THEODORO et al., 2001). Gatto et al. (2003) também constataram menor densidade do solo em tratamentos que passaram por revolvimento do solo em plantios de Eucalyptus grandis. Resultados semelhantes também foram encontrados por Reichert et al. (2009), em um Argissolo Vermelho-Amarelo distrófico típico com plantio de feijão.

Sob determinado método de preparo, as condições físicas do solo tenderão a um estado estável, o qual depende das condições edáficas e climáticas. Além disso, os diferentes métodos de preparo resultarão em diferentes condições de equilíbrio físico do solo, dependendo dos efeitos do preparo sobre a formação e estabilização dos agregados (SILVA; MIELNICZUK, 1998). Dessa forma, o estudo das propriedades físicas ao longo do tempo permite quantificar a magnitude e a duração das alterações causadas pelo manejo do solo. Por serem parâmetros sensíveis, algumas propriedades físicas são importantes para estabelecer se houve degradação ou melhoria da qualidade do solo, em relação a um sistema de manejo (REICHERT et al., 2009). Neste contexto, o objetivo do trabalho foi avaliar as alterações, após 6 anos, de propriedades físicas de um Argissolo Vermelho-Amarelo distrófico arênico e do crescimento de Eucalyptus grandis, implantado em diferentes métodos de preparo do solo. 


\section{MATERIAL E MÉTODOS}

O estudo foi realizado em uma área experimental pertencente à Fundação Estadual de Pesquisa Agropecuária (FEPAGRO), localizada no município de Santa Maria, Rio Grande do Sul (coordenadas UTM 217.000 e $220.000 \mathrm{~m} \mathrm{E}, 6.713 .000$ e $6.717 .000 \mathrm{~m} \mathrm{~N}$ - Fuso $22^{\circ} \mathrm{S}$ ). O clima da região é do tipo Cfa, conforme a classificação de Köppen, o qual corresponde ao clima subtropical úmido com verão quente (MORENO, 1961). A temperatura média anual do município de Santa Maria é de $19,2^{\circ} \mathrm{C}$, e a precipitação pluviométrica total anual é de, aproximadamente, $1708 \mathrm{~mm}$ (MALUF, 2000). O solo da área do estudo é classificado como Argissolo Vermelho-Amarelo distrófico arênico (EMBRAPA, 2013), de textura franco arenosa com, aproximadamente, $165 \mathrm{~g} \mathrm{~kg}^{-1}$ de argila, $196 \mathrm{~g} \mathrm{~kg}^{-1}$ de silte e $639 \mathrm{~g} \mathrm{~kg}^{-1}$ de areia na camada de 0-0,3 m (PREVEDELLO, 2008).

O delineamento experimental empregado foi o de blocos ao acaso com três repetições e quatro tratamentos: coveamento manual (CM), escarificado (Esc), escarificado mais grade niveladora (EG) e enxada rotativa (ER). No CM não ocorreu o revolvimento do solo, mas apenas a formação de covas de $0,10 \mathrm{~m}$ de profundidade e $0,08 \mathrm{~m}$ de diâmetro, feitas com o auxílio de um trado. O Esc foi realizado com um escarificador de uma haste, até a profundidade de 0,3 m. No tratamento EG, a escarificação foi seguida por uma passagem de grade de disco "off-set" com uma profundidade de operação de 0,1 metros. O preparo com enxada rotativa mobilizou uma faixa de 1,0 $\mathrm{m}$ de largura e 0,2 $\mathrm{m}$ de profundidade (PREVEDELLO, 2008).

O plantio do eucalipto foi feito com espaçamento de 3,0 x 2,0 m, sendo que cada unidade experimental com dimensão de $600 \mathrm{~m}^{2}$ foi composta inicialmente por 100 árvores. O preparo do solo e o plantio das mudas de Eucalyptus grandis foram realizados em novembro de 2006, sendo as mudas adquiridas da empresa Tecnoplanta Florestal LTDA, a qual as produziu por meio de sementes do pomar clonal da Rigesa. Trinta dias após o plantio foi realizada a aplicação de $218 \mathrm{~kg} \mathrm{ha}^{-1}$ de NPK fórmula 33-80-18, conforme as recomendações técnicas para o eucalipto (CQFS-RS/SC, 2004). Aos dois e aos cinco meses após o plantio foi realizada roçada mecânica na entrelinha para reduzir a matocompetição. Um mês após o plantio foi feito o replantio de $15 \%$ das mudas devido, principalmente, ao ataque de formigas e às condições climáticas adversas (PREVEDELLO, 2008).

Anteriormente à implantação do experimento, a área do estudo encontrava-se em pousio por mais de 20 anos, sem alterações antrópicas, predominando as espécies: Paspalum notatum (Grama forquilha), Andropogon lateralis (Capim caninha), Axonopus pressus (Grama missioneira) e Aristida pallens (barba-de-bode), caracterizando a vegetação do campo nativo. Previamente à aplicação dos tratamentos, a vegetação existente na área experimental foi eliminada, por meio de roçada mecanizada, e deixada sobre o solo (PREVEDELLO, 2008).

As amostras de solo foram coletadas antes da aplicação dos tratamentos e 72 meses após a aplicação dos tratamentos. Além disso, há dados disponíveis de uma coleta realizada três meses após a aplicação (Figura 2B) (PREVEDELLO, 2008). Para a determinação da densidade, macroporosidade, microporosidade e porosidade total do solo coletaram-se amostras na linha de plantio, com cilindros metálicos (0,06 m de diâmetro e 0,03 m de altura), nas camadas de $0-0,1 ; 0,1-0,2 ; 0,2-0,3$ e 0,3-0,4 m. Foi feita uma trincheira por unidade experimental, totalizando 12 pontos de coleta, sendo coletadas duas repetições por camada em cada ponto de coleta. Na camada de 0-0,05 foram coletadas amostras indeformadas, na linha de plantio, para a determinação da distribuição do tamanho dos agregados estáveis em água e amostras deformadas para a determinação da matéria orgânica do solo. Os pontos amostrais foram aproximadamente os mesmos realizados por Prevedello (2008).

A macroporosidade e a microporosidade foram obtidas pelo método da mesa de tensão com coluna d'água de $0,60 \mathrm{~m}$ e a porosidade total foi calculada pela soma dos valores de macroporosidade e microporosidade do solo (EMBRAPA, 1997). A densidade do solo foi determinada pelo método do anel volumétrico (EMBRAPA, 1997). A matéria orgânica do solo foi obtida a partir da multiplicação do teor de carbono orgânico do solo, determinado pelo método de combustão úmida (TEDESCO et al.,1995), pelo fator de 1,724.

Para a determinação da distribuição do tamanho dos agregados estáveis em água, empregou-se o método de Kemper e Chepil (1965) modificado, utilizando-se amostras de agregados menores que $8 \mathrm{~mm}$. Os agregados foram separados manualmente observando-se as superfícies naturais de clivagem e, posteriormente, umedecidos por capilaridade por 10 minutos e agitados durante 10 minutos, 
em um aparelho de oscilação vertical, com 30 oscilações por minuto (YODER, 1936). O diâmetro médio geométrico (DMG), o qual representa uma estimativa dos agregados de maior ocorrência (CASTRO FILHO et al., 1998), o diâmetro médio ponderado (DMP), o qual é tanto maior quanto maior a percentagem de agregados grandes (CASTRO FILHO et al., 1998), e a percentagem de agregados por classe de tamanho (PA), foram obtidos pelas seguintes equações:

$$
\begin{gathered}
D M P=\sum\left(M A_{i} d_{i} / M A T\right) \\
D M G=\exp \left(\sum\left(M A_{i} \ln \left(d_{i}\right) / M A T\right)\right) \\
P A_{i}=\left(M A_{i} / M A T\right) 100
\end{gathered}
$$

em que: $\mathrm{DMG}$ = diâmetro médio geométrico $(\mathrm{mm})$; $\mathrm{DMP}=$ diâmetro médio ponderado $(\mathrm{mm})$; $\mathrm{MA}_{\mathrm{i}}=$ massa de agregados da classe $\mathrm{i}(\mathrm{g}), \mathrm{MAT}=$ massa total de agregados descontada a fração inerte (g); $\mathrm{d}_{\mathrm{i}}=$ diâmetro médio da classe i $(\mathrm{mm})$.

Para avaliar o crescimento do povoamento de Eucalyptus grandis, foram realizadas medições das variáveis dendrométricas altura total e diâmetro, as quais foram feitas em todas as árvores remanescentes de cada unidade experimental (28 árvores, aproximadamente), exceto nas árvores deixadas como bordadura. A bordadura correspondeu a duas linhas de árvores em cada lado de cada uma das unidades experimentais.

Até os 12 meses após o preparo do solo, a altura total e o diâmetro a $10 \mathrm{~cm}$ de altura das árvores $\left(\mathrm{d}_{10}\right)$ foram medidos com o uso de régua telescópica e paquímetro digital, respectivamente (PREVEDELO, 2008). Aos 74 meses após o preparo do solo foram feitas medições do perímetro à altura do peito ( $1,3 \mathrm{~m}$ do nível do solo) e das alturas totais das árvores com o uso de fita métrica e hipsômetro Vertex IV, respectivamente. Posteriormente, o perímetro à altura do peito foi convertido em diâmetro à altura do peito (DAP). Os dados de solo e de crescimento até os 12 meses após a aplicação dos tratamentos foram obtidos por Prevedello (2008).

Após ser atendido o pressuposto de homogeneidade de variâncias e normalidade dos erros, os dados foram submetidos à análise de variância e, quando o teste $\mathrm{F}$ foi significativo, as médias dos tratamentos foram comparadas pelo teste de Tukey $(\mathrm{p}<0,05)$. Foram testados os efeitos do método de preparo do solo, da época de avaliação e da interação entre ambos no conteúdo de matéria orgânica e nas propriedades físicas do solo, sendo realizada uma análise de variância por camada. Na análise de dados de diâmetro, altura e sobrevivência das árvores foi calculada análise de variância com um fator (preparo do solo) para cada época.

\section{RESULTADOS E DISCUSSÃO}

\section{Propriedades físicas e matéria orgânica do solo}

Para nenhuma das variáveis de solo avaliadas houve interação entre métodos de preparo do solo e época, logo, apenas os efeitos principais foram analisados. Para DMG e DMP não ocorreu diferença significativa entre tratamentos e, aos 72 meses após o preparo do solo os valores médios de DMG e DMP não diferiram dos valores médios ocorridos antes do preparo do solo (Tabela 1). Com base nestes resultados e tendo em vista o revolvimento e a desestruturação do solo provocado pelos tratamentos Esc, EG e ER, que alteraram significativamente a estrutura do solo logo após seu preparo (PREVEDELLO, 2008), verificou-se nesses tratamentos uma recuperação da agregação aos 72 meses após o revolvimento do solo.

A recuperação da estrutura do solo, aos 72 meses após o preparo, é um provável reflexo do desenvolvimento e crescimento do sistema radicular do eucalipto, assim como da atividade dos organismos do solo. As raízes finas das árvores, assim como as hifas de fungos, formam uma rede que entremeia microagregados (diâmetro $<250 \mu \mathrm{m}$ ) e pequenos macroagregados, formando e estabilizando grandes macroagregados (diâmetro >2000 $\mu \mathrm{m}$ ) (TISDALL; OADES, 1982). De acordo com Zonta et al. (2006), a pressão exercida pelo crescimento radicular promove a aproximação das partículas do solo. Da mesma forma, a absorção de água pelas raízes também favorece a agregação pelo secamento na região adjacente às raízes, causando um aumento na força de coesão entre as 
partículas minerais do solo. Além disso, as mucilagens exsudadas pelas raízes, fungos e bactérias edáficos envolvem as partículas do solo atuando como agentes cimentantes, promovendo a estruturação e aumento da estabilidade dos agregados (ROZANE et al., 2010; ZINN et al., 2011; BRANDÃO; SILVA, 2012).

Tabela 1. Diâmetro médio geométrico (DMG), diâmetro médio ponderado (DMP) e teor de matéria orgânica do solo (MOS) na camada de 0-0,05 m de solo cultivado com Eucalyptus grandis, para os tratamentos: coveamento manual (CM), escarificado (Esc), escarificado mais grade niveladora (EG) e enxada rotativa (ER), em Santa Maria, RS.

Table 1. Geometric mean diameter (DMG), mean weight diameter (DMP) and organic matter content (MOS) of the 0-0,05 m soil layer cultivated with Eucalyptus grandis, for the treatments: manual hoeing (CM), chisel plow (Esc), chisel plow plus harrowing (EG) and rotary hoe (ER), in Santa Maria, RS.

\begin{tabular}{|c|c|c|c|c|c|}
\hline \multirow{2}{*}{ Época* } & \multicolumn{4}{|c|}{ Métodos de preparo do solo } & \multirow{2}{*}{ Média } \\
\hline & CM & Esc & EG & ER & \\
\hline & \multicolumn{5}{|c|}{ DMG (mm) } \\
\hline Antes do preparo & $2,19(0,129)$ & $2,57 \quad(0,503)$ & $2,52(0,203)$ & $2,54 \quad(0,558)$ & $2,45 a^{* *}$ \\
\hline 72 meses após o preparo & $2,53(0,426)$ & $2,47 \quad(0,674)$ & $2,12(0,305)$ & $2,61 \quad(0,568)$ & 2,43 a \\
\hline \multirow[t]{2}{*}{ Média } & $2,36 \mathrm{~A}$ & $2,52 \mathrm{~A}$ & $2,32 \mathrm{~A}$ & $2,58 \mathrm{~A}$ & \\
\hline & \multicolumn{5}{|c|}{ DMP (mm) } \\
\hline Antes do preparo & $3,75 \quad(0,178)$ & $4,13(0,393)$ & $4,02(0,161)$ & $4,16(0,502)$ & 4,02 a \\
\hline 72 meses após o preparo & $4,14 \quad(0,358)$ & $4,02(0,560)$ & $3,66 \quad(0,347)$ & $4,11 \quad(0,440)$ & 3,98 a \\
\hline \multirow[t]{2}{*}{ Média } & $3,94 \mathrm{~A}$ & $4,07 \mathrm{~A}$ & $3,84 \mathrm{~A}$ & $4,14 \mathrm{~A}$ & \\
\hline & \multicolumn{5}{|c|}{ MOS (\%) } \\
\hline Antes do preparo & $2,02 \quad(0,409)$ & $1,98 \quad(0,175)$ & $1,84(0,395)$ & $1,81 \quad(0,244)$ & $1,91 b$ \\
\hline 72 meses após o preparo & $2,30 \quad(0,322)$ & $2,07 \quad(0,366)$ & $2,22(0,584)$ & $2,26(0,514)$ & 2,21 a \\
\hline Média & $2,16 \mathrm{~A}$ & $2,02 \mathrm{~A}$ & $2,03 \mathrm{~A}$ & $2,03 \mathrm{~A}$ & \\
\hline
\end{tabular}

Valores entre parênteses correspondem ao desvio-padrão.

* Os dados obtidos antes do preparo do solo foram coletados por Prevedello (2008).

***édias seguidas de letras iguais, maiúsculas na linha e minúsculas na coluna, não diferem estatisticamente entre si pelo Teste de Tukey ( $p<0,05$ ) $(n=12)$.

O conteúdo de matéria orgânica do solo aos 72 meses após o preparo do solo também não diferiu entre tratamentos, contudo, foi maior do que o conteúdo encontrado antes do preparo do solo (Tabela 1). O maior conteúdo de matéria orgânica do solo aos 72 meses também pode ter contribuído para o aumento da agregação e melhoria da estrutura do solo nesse período de tempo, haja vista que, como reportam Zinn et al. (2011), a matéria orgânica do solo tem ação cimentante, atuando sobre a estabilização dos agregados.

O maior conteúdo de matéria orgânica aos 72 meses após o preparo do solo pode ser resultado do acúmulo de serapilheira proporcionado pelo eucalipto, o qual provavelmente seja maior no plantio de eucalipto aos 72 meses de idade do que no campo nativo. Por exemplo, Montero (2008) encontrou maiores valores de serapilheira para plantios de eucalipto (11,71 t.ha ${ }^{-1}$, em média), com idade superior a 30 anos, do que para remanescentes de cerradão $\left(9,18\right.$ t.ha $^{-1}$, em média). Mendham et al. (2004) observaram efeito significativo da natureza física e química da serapilheira de Eucalyptus globulus sobre a concentração de carbono da matéria orgânica particulada, ao comparar com pastagem. Mendham et al. (2004) encontraram uma concentração de $75 \%$ no plantio de eucalipto de 11 a 14 anos e 62\% em área com pastagens estabelecidas há 30 a 74 anos. O tipo de serapilheira produzida no plantio de eucalipto pode também explicar o resultado observado (Tabela $1)$, pois provavelmente difere da serapilheira original.

Para a distribuição do tamanho dos agregados estáveis em água, houve diferença significativa entre as duas épocas estudadas somente nas classes de 1,00-0,25 e <0,25 mm (Figura 1). Acima da classe de 2,00-1,00 $\mathrm{mm}$ as percentagens de agregados estáveis em água das duas épocas de coleta não diferiram entre si. Nas duas épocas avaliadas houve maior percentagem de agregados estáveis em água na classe de 8,00-4,76 mm, o que é favorável para a estrutura do solo, visto que agregados maiores e mais estáveis são mais resistentes ao processo erosivo (BERTONI; LOMBARDI NETO, 2008). Como complementam Castro Filho et al. (1998), quanto maior for o tamanho do agregado, maiores serão o DMP e os espaços porosos entre agregados, aumentando a infiltração e diminuindo a erosão. 
Rocha et al. - Propriedades físicas do solo e crescimento de

eucalipto implantado em diferentes métodos de preparo do solo

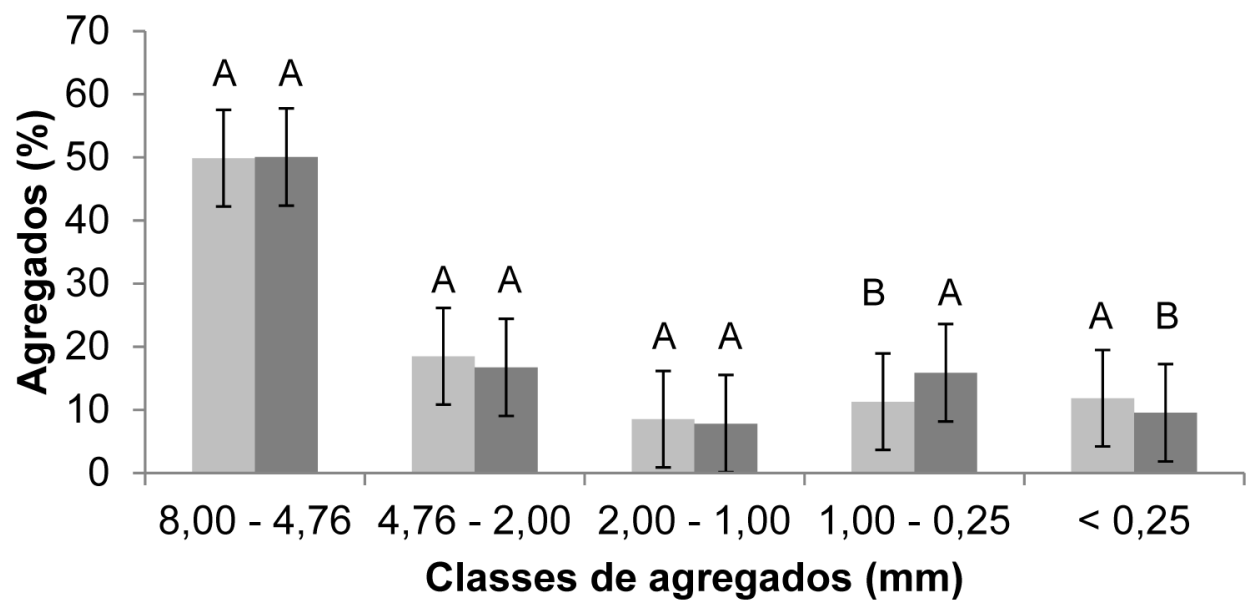

Antes do preparo do solo $\square 72$ meses após o preparo do solo

Figura 1. Distribuição do tamanho dos agregados estáveis em água na camada de 0-0,05 m, de um Argissolo Vermelho-Amarelo distrófico arênico cultivado com Eucalyptus grandis, em Santa Maria, RS. Médias seguidas de letras iguais na mesma classe de agregados não diferem entre si pelo Teste de Tukey $(p<0,05)$. Barras verticais representam o erro padrão $(n=12)$.

Figure 1. Size distribution of water-stable aggregates at a depth of 0-0.05 m, of a Alfisol cultivated with Eucalyptus grandis, in Santa Maria, RS. Means followed by the same letters not differ by the Tukey $(p<0,05)$. Vertical bars represent the standard error $(n=12)$.

Em todas as camadas avaliadas não foi observada diferença significativa entre os tratamentos para densidade, macroporosidade (Tabela 2), microporosidade e porosidade total do solo (Tabela 3 ), diferentemente do observado por Prevedello (2008) logo após o preparo do solo. Três meses após o preparo do solo, Prevedello (2008) encontrou menores valores de macroporosidade e porosidade total e maiores valores de densidade do solo para o tratamento CM, provavelmente devido à consolidação natural do solo em função da ausência de preparo nesse tratamento (Figura 2). Conforme Tormena et al. (2002), o revolvimento do solo provoca o fraturamento dos agregados e o desenvolvimento de poros, notadamente macroporos, causando a redução da densidade do solo e da resistência à penetração das raízes.
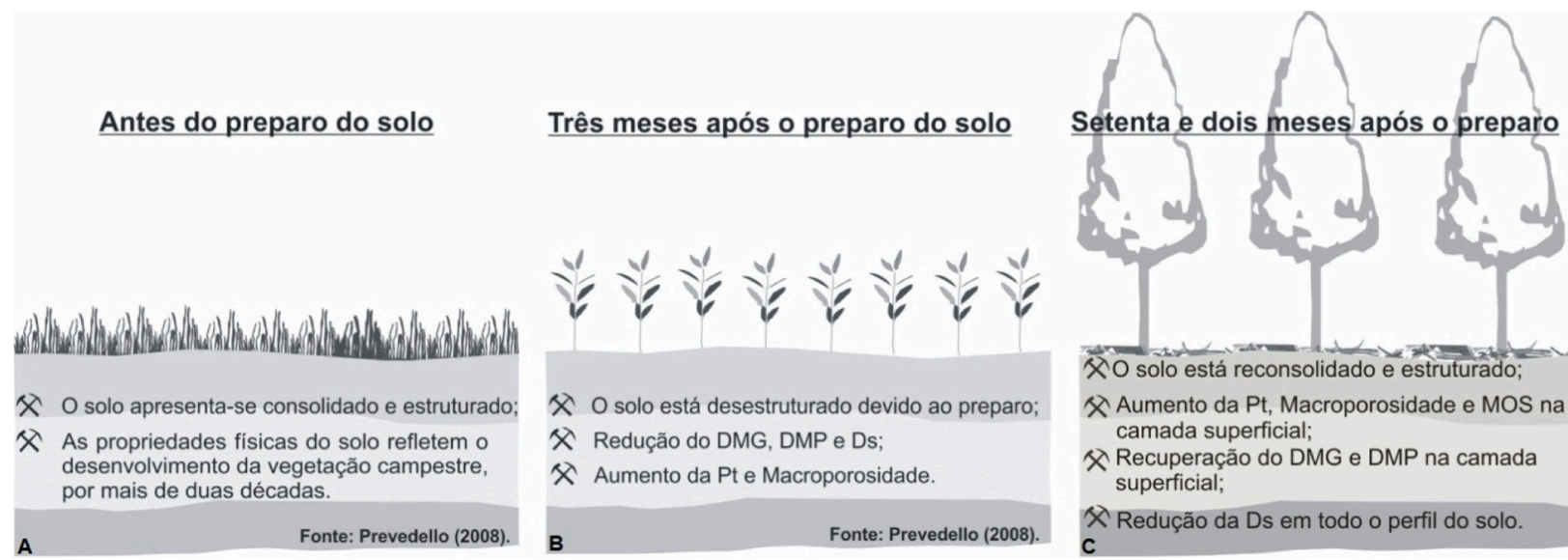

X Redução do DMG, DMP e Ds;

X Aumento da Pt e Macroporosidade.
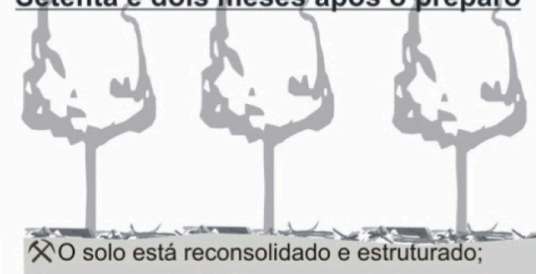

A Aumento da Pt, Macroporosidade e MOS na camada superficial; Recuperação do DMG e DMP na camada
superficial;

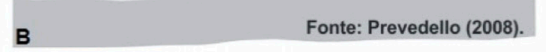

X' Redução da Ds em todo o perfil do solo.

Figura 2. Propriedades físicas de um Argissolo Vermelho-Amarelo distrófico arênico cultivado com Eucalyptus grandis, em Santa Maria, RS, antes do preparo do solo (A) e após três (B) e 72 meses de plantio (C). DMG = Diâmetro Médio Geométrico. DMP = Diâmetro Médio Ponderado. Ds = densidade do solo. Pt $=$ porosidade total. MOS = matéria orgânica do solo. 2B corresponde aos tratamentos com revolvimento do solo.

Figure 2. Physical properties of an Alfisol cultivated with Eucalyptus grandis, in Santa Maria, RS, before (A) the tillage and after three (B) and 72 months of planting (C). DMG = Medium Geometric Diameter. DMP = mean weight diameter. $\mathrm{Ds}=$ bulk density. $\mathrm{Pt}=$ total porosity. MOS = soil organic matter. $2 \mathrm{~B}$ corresponds to the treatments with soil tillage.

Os valores de densidade do solo variaram de 1,23 a 1,57 $\mathrm{g} \mathrm{cm}^{-3}$ (Tabela 2), ficando abaixo dos valores considerados restritivos ao crescimento radicular para solos franco-arenosos para culturas agrícolas, conforme Reichert et al. (2003), que é de 1,70 - 1,80 $\mathrm{g} \mathrm{m}^{-3}$. As médias de densidade do 
solo antes e aos 72 meses após o preparo do solo diferiram entre si, em todas as camadas avaliadas, com menores valores aos 72 meses (Tabela 2). A redução da densidade do solo aos 72 meses após o preparo pode indicar melhoria da qualidade física do solo, decorrente possivelmente da atividade da fauna edáfica e das raízes das árvores, atuando na formação de bioporos e na estabilização da estrutura do solo (LAURINDO et al., 2009), principalmente nas camadas superficiais, onde há maior densidade de raízes e maior atividade biológica. Em relação à densidade de raízes, Silva et al. (2009) observaram maior densidade de raízes finas (drf) até $30 \mathrm{~cm}$ de profundidade, aproximadamente, mas com presença de raízes finas até 2 metros, em povoamento de Eucalyptus grandis de 6, 12, 18 e 30 meses de idade.

Tabela 2. Densidade e macroporosidade do solo cultivado com Eucalyptus grandis, para os tratamentos: coveamento manual (CM), escarificado (Esc), escarificado mais grade niveladora (EG) e enxada rotativa (ER), em Santa Maria, RS.

Table 2. Bulk density and macroporosity of the soil cultivated with Eucalyptus grandis, for the treatments: manual hoeing (CM), chisel plow (Esc), chisel plow plus harrowing (EG) and rotary hoe (ER), in Santa Maria, RS.

\begin{tabular}{|c|c|c|c|c|c|c|c|}
\hline \multirow{2}{*}{ Época* } & \multicolumn{6}{|c|}{ Métodos de preparo do solo } & \multirow{2}{*}{ Média } \\
\hline & $\mathbf{C M}$ & Esc & & EG & & ER & \\
\hline \multicolumn{8}{|c|}{ Densidade do solo $\left(\mathrm{g} \mathrm{cm}^{-3}\right)$} \\
\hline \multicolumn{8}{|c|}{ Camada de 0-0,1 m } \\
\hline Antes do preparo & $1,52(0,034)$ & $1,48 \quad(0,021)$ & 1,43 & $(0,100)$ & 1,45 & $(0,012)$ & $1,47 a^{* *}$ \\
\hline 72 meses após o preparo & $1,38(0,109)$ & $1,24 \quad(0,037)$ & 1,32 & $(0,056)$ & 1,23 & $(0,014)$ & $1,30 \mathrm{~b}$ \\
\hline Média & $1,45 \mathrm{~A}$ & $1,36 \mathrm{~A}$ & 1,38 & $\mathrm{~A}$ & 1,34 & $\mathrm{~A}$ & \\
\hline \multicolumn{8}{|c|}{ Camada de 0,1-0,2 m } \\
\hline Antes do preparo & $1,57(0,001)$ & $1,56(0,033)$ & 1,53 & $(0,028)$ & 1,53 & $(0,021)$ & 1,55 a \\
\hline 72 meses após o preparo & $1,43(0,088)$ & $1,32(0,025)$ & 1,38 & $(0,133)$ & 1,46 & $(0,057)$ & $1,40 \quad b$ \\
\hline Média & $1,50 \mathrm{~A}$ & $1,44 \mathrm{~A}$ & 1,46 & $\mathrm{~A}$ & 1,49 & $\mathrm{~A}$ & \\
\hline \multicolumn{8}{|c|}{ Camada de $0,2-0,3 \mathrm{~m}$} \\
\hline Antes do preparo & $1,53(0,081)$ & $1,54(0,025)$ & 1,50 & $(0,031)$ & 1,48 & $(0,035)$ & 1,51 a \\
\hline 72 meses após o preparo & $1,47 \quad(0,092)$ & $1,43(0,064)$ & 1,41 & $(0,138)$ & 1,44 & $(0,043)$ & $1,44 \quad b$ \\
\hline Média & $1,50 \mathrm{~A}$ & $1,48 \mathrm{~A}$ & 1,46 & $\mathrm{~A}$ & 1,46 & $\mathrm{~A}$ & \\
\hline \multicolumn{8}{|c|}{ Camada de 0,3-0,4 m } \\
\hline Antes do preparo & $1,57 \quad(0,105)$ & $1,56(0,029)$ & 1,52 & $(0,036)$ & 1,53 & $(0,062)$ & $1,54 \quad a$ \\
\hline 72 meses após o preparo & $1,52(0,002)$ & $1,45(0,101)$ & 1,42 & $(0,093)$ & 1,48 & $(0,040)$ & $1,47 \quad b$ \\
\hline Média & $1,54 \mathrm{~A}$ & $1,51 \mathrm{~A}$ & 1,47 & $\mathrm{~A}$ & 1,50 & $\mathrm{~A}$ & \\
\hline \multicolumn{8}{|c|}{ Macroporosidade do solo $\left(\mathrm{m}^{3} \mathrm{~m}^{-3}\right)$} \\
\hline \multicolumn{8}{|c|}{ Camada de $0-0,1 \mathrm{~m}$} \\
\hline Antes do preparo & $0,10(0,024)$ & $0,11 \quad(0,020)$ & 0,14 & $(0,014)$ & 0,14 & $(0,053)$ & $0,12 \quad b$ \\
\hline 72 meses após o preparo & $0,22(0,029)$ & $0,25(0,016)$ & 0,23 & $(0,055)$ & 0,30 & $(0,000)$ & 0,25 a \\
\hline Média & $0,16 \mathrm{~A}$ & $0,18 \mathrm{~A}$ & 0,20 & $\mathrm{~A}$ & 0,22 & $\mathrm{~A}$ & \\
\hline \multicolumn{8}{|c|}{ Camada de 0,1-0,2 m } \\
\hline Antes do preparo & $0,12(0,030)$ & $0,10(0,034)$ & 0,14 & $(0,033)$ & 0,13 & $(0,024)$ & $0,12 \quad b$ \\
\hline 72 meses após o preparo & $0,13(0,018)$ & $0,19(0,028)$ & 0,18 & $(0,063)$ & 0,17 & $(0,018)$ & 0,17 a \\
\hline Média & $0,12 \mathrm{~A}$ & $0,14 \mathrm{~A}$ & 0,16 & $\mathrm{~A}$ & 0,15 & $\mathrm{~A}$ & \\
\hline \multicolumn{8}{|c|}{ Camada de 0,2-0,3 m } \\
\hline Antes do preparo & $0,11 \quad(0,044)$ & $0,10 \quad(0,040)$ & 0,14 & $(0,035)$ & 0,14 & $(0,036)$ & 0,12 a \\
\hline 72 meses após o preparo & $0,14 \quad(0,047)$ & $0,15 \quad(0,007)$ & 0,16 & $(0,101)$ & 0,16 & $(0,030)$ & 0,15 a \\
\hline Média & $0,12 \mathrm{~A}$ & $0,12 \mathrm{~A}$ & 0,15 & $\mathrm{~A}$ & 0,15 & $\mathrm{~A}$ & \\
\hline \multicolumn{8}{|c|}{ Camada de 0,3-0,4 m } \\
\hline Antes do preparo & $0,10 \quad(0,053)$ & $0,09 \quad(0,023)$ & 0,12 & $(0,024)$ & 0,11 & $(0,029)$ & 0,11 a \\
\hline 72 meses após o preparo & $0,12(0,045)$ & $0,12(0,020)$ & 0,15 & $(0,063)$ & 0,13 & $(0,021)$ & 0,13 a \\
\hline Média & $0,11 \mathrm{~A}$ & $0,11 \mathrm{~A}$ & 0,14 & A & 0,12 & A & \\
\hline
\end{tabular}

Os valores entre parênteses correspondem ao desvio-padrão. * Os dados obtidos antes do preparo do solo foram coletados por Prevedello (2008). ***édias seguidas de letras iguais, maiúsculas na linha e minúsculas na coluna, não diferem estatisticamente entre si pelo Teste de Tukey ( $p<0,05$ ) $(n=12)$.

O maior conteúdo de matéria orgânica do solo aos 72 meses após o preparo também pode ser listado como um dos fatores responsáveis pelos menores valores de densidade do solo neste período. A matéria orgânica age na floculação do solo, proporcionando maior macroporosidade e, por isso, diminui a relação massa volume, causando a redução da densidade do solo (LAURINDO et al., 
2009). Dessa forma, como a densidade do solo é um atributo que reflete o arranjo das partículas do solo, ela está intimamente associada às características do sistema poroso (FERREIRA, 2010).

A macroporosidade e a porosidade total do solo aumentaram aos 72 meses após o preparo na camada superficial do solo. As médias de macroporosidade diferiram entre antes e 72 meses após o preparo do solo nas camadas de 0-0,10 e 0,10-0,20 m (Tabela 2). Em todos os tratamentos avaliados, os valores de macroporosidade aos 72 meses após o preparo do solo foram maiores que o valor limite considerado crítico para o crescimento satisfatório das plantas, proposto por Xu et al. (1992), que é de $0,10 \mathrm{~m}^{3} \mathrm{~m}^{-3}$. Em contrapartida, antes da implantação do experimento foram observados valores abaixo ou iguais ao valor considerado crítico. Já para a porosidade total, ocorreu aumento significativo entre os dois períodos em estudo apenas na camada de 0-0,10 m (Tabela 3).

Tabela 3. Microporosidade e porosidade total do solo cultivado com Eucalyptus grandis, para os tratamentos: coveamento manual (CM), escarificado (Esc), escarificado mais grade niveladora (EG) e enxada rotativa (ER), em Santa Maria, RS.

Table 3. Micro porosity and total porosity of the soil cultivated with Eucalyptus grandis, for the treatments: manual hoeing (CM), chisel plow (ESC), chisel plow plus harrowing (EG) and rotary hoe (ER), in Santa Maria, RS.

\begin{tabular}{|c|c|c|c|c|c|c|c|}
\hline \multirow{2}{*}{ Época* } & \multicolumn{5}{|c|}{ Métodos de preparo do solo } & \multirow{2}{*}{ Média } & \\
\hline & CM & Esc & & EG & ER & & \\
\hline \multicolumn{7}{|c|}{ Microporosidade do solo $\left(\mathrm{m}^{3} \mathrm{~m}^{-3}\right)$} & \\
\hline \multicolumn{8}{|c|}{ Camada de $0-0,1 \mathrm{~m}$} \\
\hline Antes do preparo & $0,31 \quad(0,034)$ & $0,31 \quad(0,006)$ & 0,30 & $(0,034)$ & $0,28 \quad(0,040)$ & $0,30 a^{*}$ & $a^{* *}$ \\
\hline 72 meses após o preparo & $0,24 \quad(0,025)$ & $0,22(0,004)$ & 0,21 & $(0,017)$ & $0,23(0,005)$ & $0,22 \mathrm{~b}$ & b \\
\hline Média & $0,28 \mathrm{~A}$ & $0,27 \mathrm{~A}$ & 0,26 & A & $0,25 \mathrm{~A}$ & & \\
\hline \multicolumn{8}{|c|}{ Camada de 0,1-0,2 m } \\
\hline Antes do preparo & $0,27(0,006)$ & $0,27 \quad(0,018)$ & 0,25 & $(0,020)$ & $0,25(0,013)$ & 0,26 & $\bar{a}$ \\
\hline 72 meses após o preparo & $0,25(0,018)$ & $0,24 \quad(0,015)$ & 0,23 & $(0,003)$ & $0,23(0,011)$ & 0,23 & $b$ \\
\hline Média & $0,26 \mathrm{~A}$ & $0,25 \mathrm{~A}$ & 0,24 & A & $0,24 \mathrm{~A}$ & & \\
\hline \multicolumn{8}{|c|}{ Camada de 0,2-0,3 m } \\
\hline Antes do preparo & $0,26(0,020)$ & $0,26(0,023)$ & 0,26 & $(0,006)$ & $0,24 \quad(0,005)$ & 0,26 & \\
\hline 72 meses após o preparo & $0,25(0,019)$ & $0,24 \quad(0,007)$ & 0,23 & $(0,008)$ & $0,23(0,007)$ & 0,24 & $b$ \\
\hline Média & $0,25 \mathrm{~A}$ & $0,25 \mathrm{~A}$ & 0,25 & $\mathrm{~A}$ & $0,24 \mathrm{~A}$ & & \\
\hline \multicolumn{8}{|c|}{ Camada de 0,3-0,4 m } \\
\hline Antes do preparo & $0,27(0,018)$ & $0,27 \quad(0,009)$ & 0,25 & $(0,004)$ & $0,25 \quad(0,005)$ & 0,26 & $\bar{a}$ \\
\hline 72 meses após o preparo & $0,25(0,017)$ & $0,29(0,076)$ & 0,24 & $(0,005)$ & $0,24 \quad(0,007)$ & 0,25 & a \\
\hline Média & $0,26 \mathrm{~A}$ & $0,28 \mathrm{~A}$ & 0,25 & $\mathrm{~A}$ & $0,24 \mathrm{~A}$ & & \\
\hline \multicolumn{8}{|c|}{ Porosidade total do solo $\left(\mathrm{m}^{3} \mathrm{~m}^{-3}\right)$} \\
\hline \multicolumn{8}{|c|}{ Camada de $0-0,1 \mathrm{~m}$} \\
\hline Antes do preparo & $0,41 \quad(0,025)$ & $0,42(0,014)$ & 0,44 & $(0,022)$ & $0,42(0,049)$ & 0,42 & $\mathrm{~b}$ \\
\hline 72 meses após o preparo & $0,46 \quad(0,050)$ & $0,46(0,014)$ & 0,47 & $(0,072)$ & $0,52(0,005)$ & 0,48 & a \\
\hline Média & $0,43 \mathrm{~A}$ & $0,44 \quad \mathrm{~A}$ & 0,46 & $\mathrm{~A}$ & $0,47 \mathrm{~A}$ & & \\
\hline \multicolumn{8}{|c|}{ Camada de 0,1-0,2 m } \\
\hline Antes do preparo & $0,38(0,024)$ & $0,37(0,017)$ & 0,39 & $(0,039)$ & $0,38 \quad(0,016)$ & 0,38 & $a$ \\
\hline 72 meses após o preparo & $0,38(0,001)$ & $0,43(0,013)$ & 0,41 & $(0,063)$ & $0,39(0,019)$ & 0,40 & a \\
\hline Média & $0,38 \quad A$ & $0,40 \quad \mathrm{~A}$ & 0,40 & A & $0,38 \mathrm{~A}$ & & \\
\hline \multicolumn{8}{|c|}{ Camada de $0,2-0,3 \mathrm{~m}$} \\
\hline Antes do preparo & $0,37(0,028)$ & $0,36(0,018)$ & 0,41 & $(0,038)$ & $0,38(0,041)$ & 0,38 & \\
\hline 72 meses após o preparo & $0,39(0,043)$ & $0,39(0,010)$ & 0,39 & $(0,093)$ & $0,39(0,036)$ & 0,39 & a \\
\hline Média & $0,38 \mathrm{~A}$ & $0,38 \quad \mathrm{~A}$ & 0,40 & $\mathrm{~A}$ & $0,39 \mathrm{~A}$ & & \\
\hline \multicolumn{8}{|c|}{ Camada de 0,3-0,4 m } \\
\hline Antes do preparo & $0,36(0,039)$ & $0,36(0,014)$ & 0,38 & $(0,026)$ & $0,36(0,031)$ & 0,37 & \\
\hline 72 meses após o preparo & $0,37 \quad(0,058)$ & $0,41 \quad(0,071)$ & 0,38 & $(0,059)$ & $0,36(0,019)$ & 0,38 a & \\
\hline Média & $0,37 \mathrm{~A}$ & $0,38 \mathrm{~A}$ & 0,38 & A & $0,36 \mathrm{~A}$ & & \\
\hline
\end{tabular}


Além do maior aporte de matéria orgânica na superfície do solo, proveniente da serapilheira do eucalipto e da decomposição da vegetação campestre anterior à implantação do experimento, o sistema radicular do eucalipto também pode ter contribuído para o aumento da macroporosidade e porosidade total do solo aos 72 meses após o preparo do solo. Como evidenciado no estudo de Silva et al. (2009), a maior densidade de raízes do eucalipto ocorre na superfície do solo e, como reportam Brady e Weil (2013), a morte e decomposição das raízes formam os bioporos, sendo as árvores de florestas particularmente eficientes na formação destes poros.

Contrariamente à macroporosidade e a porosidade total do solo, a microporosidade diminuiu ao longo dos anos até $0,30 \mathrm{~m}$ de profundidade do solo. Todavia, na camada de 0,30-0,40 m não ocorreu diferença entre as duas épocas avaliadas (Tabela 3).

Tendo em vista as alterações causadas nas propriedades físicas e no conteúdo de matéria orgânica do solo logo após seu preparo (PREVEDELLO, 2008), e considerando o fato de que as médias destas propriedades aos 72 meses após o preparo foram significativamente iguais ou maiores do que as médias antes do preparo (Figura 2), pode-se inferir que o solo se reconsolidou, retornando a uma condição estrutural semelhante ou melhor que a condição inicial (Figura 2). Semelhante ao presente estudo, Paes et al. (2013) observaram efeito insignificante do preparo do solo em plantio de Eucalyptus grandis x E. urophylla com 83 meses de idade, resultante da reconsolidação do solo. Reichert et al. (2009) observaram que os efeitos da escarificação sobre a densidade do solo e a resistência à penetração das raízes desapareceram depois de um ano do preparo de um Argissolo Vermelho-Amarelo com cultura agrícola. Já Rosa et al. (2008) verificaram que os efeitos da escarificação perduraram por mais de dois anos em um Latossolo Vermelho distrófico com cultura agrícola.

A redução da densidade e o aumento da porosidade e da matéria orgânica do solo aos 72 meses após o preparo do solo, observados no presente estudo, podem ter contribuído para a melhoria da qualidade edáfica, favorecendo o crescimento radicular, assim como podem ter causado a melhoria da infiltração de água e da movimentação de nutrientes e gases no perfil do solo. Da mesma forma, Mishra et al. (2003) avaliaram plantios de Eucalyptus tereticornis de 3, 6 e 9 anos de idade e encontraram maior taxa de infiltração e permeabilidade do solo no plantio com 9 anos, fato justificado pelos autores pela melhoria as condições do solo pelo incremento da porosidade, da capacidade de retenção de água e da redução densidade do solo.

\section{Crescimento do povoamento de Eucalyptus grandis}

Até os 12 meses de idade do povoamento de eucalipto registrou-se diferença significativa entre os tratamentos para altura, DAP e sobrevivência das árvores, apresentando, na maioria dos casos, maiores valores nos tratamentos em que houve revolvimento do solo (Tabela 4). Gonçalves e Stape (2002) citam que o preparo do solo causa o aumento do espaço poroso e promove a redução da densidade do solo, favorecendo a movimentação de água e as trocas gasosas no sistema solo-planta-atmosfera, resultando em melhores condições para o estabelecimento e crescimento das plantas. Avaliando o efeito da subsolagem em um Argissolo sobre o crescimento de Eucalyptus grandis, Finger et al. (1996) constataram que o crescimento das plantas em solo subsolado foi superior em mais de $50 \%$ para o DAP e o crescimento em altura das árvores em solo subsolado foi superior a 35\%, alcançando 62\% na idade de 24 meses. Efeito semelhante foi encontrado por Baptista e Levien (2010), os quais verificaram que aos 11 meses de idade de Eucalyptus saligna a biomassa aérea foi cinco vezes maior no tratamento com subsolagem em relação ao coveamento mecânico.

Aos 74 meses após o preparo do solo, não foi observada diferença significativa do DAP e da altura das árvores entre os tratamentos do presente estudo (Tabela 4). De acordo com Gonçalves (1995), apesar de o crescimento inicial de florestas de eucalipto estabelecidas no sistema de cultivo mínimo do solo geralmente ser mais lento e heterogêneo, o vigor e a homogeneidade dos povoamentos se restabelecem alguns meses após o plantio, dependendo da qualidade do sítio, o que pode explicar os resultados do presente estudo. Corroborando com o presente estudo, González et al. (2012) não encontraram diferença de crescimento em DAP de Eucalyptus grandis entre sistemas de manejo com e sem revolvimento do solo nas idades de 21, 38, 58, 98 e 120 meses. 
Tabela 4. Diâmetro a $10 \mathrm{~cm}$ de altura, diâmetro altura do peito (1,3 m acima do nível do solo), altura total e sobrevivência de Eucalyptus grandis, para os tratamentos: coveamento manual (CM), escarificado (Esc), escarificado mais grade niveladora (EG) e enxada rotativa (ER), em Santa Maria, RS.

Table 4. Diameter at $10 \mathrm{~cm}$ of height, diameter at breast height ( $1.3 \mathrm{~m}$ above the ground level), total height and survival of Eucalyptus grandis, for the treatments: manual hoeing (CM), chisel plow (Esc), chisel plow plus harrowing (EG) and rotary hoe (ER), in Santa Maria, RS.

\begin{tabular}{|c|c|c|c|c|}
\hline \multirow{2}{*}{ Época (meses)* } & \multicolumn{4}{|c|}{ Métodos de preparo do solo } \\
\hline & CM & Esc & EG & ER \\
\hline \multicolumn{5}{|c|}{ Diâmetro a $10 \mathrm{~cm}$ de altura da árvore $(\mathrm{cm})$} \\
\hline 3 & $1,14 \mathrm{~B}^{* *}$ & $1,30 \mathrm{AB}$ & $1,31 \mathrm{AB}$ & $1,47 \mathrm{~A}$ \\
\hline 6 & $2,92 \mathrm{~B}$ & $2,88 \mathrm{~B}$ & $3,03 \mathrm{~B}$ & $3,47 \mathrm{~A}$ \\
\hline 9 & $3,26 \mathrm{~B}$ & $3,24 \mathrm{~B}$ & $3,38 \mathrm{~B}$ & $4,00 \mathrm{~A}$ \\
\hline \multicolumn{5}{|c|}{ Diâmetro à altura do peito $(\mathrm{cm})$} \\
\hline 12 & $1,57 \mathrm{~B}$ & $1,95 \mathrm{AB}$ & $2,06 \mathrm{AB}$ & $2,69 \mathrm{~A}$ \\
\hline 74 & $16,14 \mathrm{~A}$ & $15,93 \mathrm{~A}$ & $15,70 \mathrm{~A}$ & $16,74 \mathrm{~A}$ \\
\hline \multicolumn{5}{|c|}{ Altura total $(\mathrm{m})$} \\
\hline 3 & $0,80 \mathrm{~B}$ & $1,03 \mathrm{~A}$ & $1,01 \mathrm{~A}$ & $1,07 \mathrm{~A}$ \\
\hline 6 & $1,83 \mathrm{~B}$ & $1,95 \mathrm{~B}$ & $1,99 \mathrm{AB}$ & $2,17 \mathrm{~A}$ \\
\hline 9 & $1,87 \mathrm{~B}$ & $2,06 \mathrm{~B}$ & $2,04 \mathrm{~B}$ & $2,29 \mathrm{~A}$ \\
\hline 12 & $2,40 \mathrm{~B}$ & $2,66 \mathrm{~B}$ & $2,80 \mathrm{~B}$ & $3,58 \mathrm{~A}$ \\
\hline 74 & $21,05 \mathrm{~A}$ & $21,73 \mathrm{~A}$ & $21,34 \mathrm{~A}$ & $22,71 \mathrm{~A}$ \\
\hline \multicolumn{5}{|c|}{ Sobrevivência (\%) } \\
\hline 12 & $59 \mathrm{C}$ & $82 \mathrm{AB}$ & $87 \mathrm{AB}$ & $91 \mathrm{~A}$ \\
\hline 74 & $59 \mathrm{~B}$ & $81 \mathrm{~A}$ & $82 \mathrm{~A}$ & $81 \mathrm{~A}$ \\
\hline
\end{tabular}

*Dados de diâmetro, altura e sobrevivência até os 12 meses de idade das árvores foram obtidos por Prevedello (2008). **Médias seguidas de letras iguais na linha não diferem estatisticamente entre si pelo Teste de Tukey $(p<0,05)$.

Para sobrevivência das árvores foi constatada diferença significativa entre os tratamentos, com sobrevivência de, aproximadamente, $20 \%$ menor no CM comparando com os demais tratamentos (Tabela 4). Com base nesse resultado, o CM pode não ser recomendado para plantios nas mesmas condições climáticas e de sítio do presente estudo.

\section{CONCLUSÕES}

Decorridos 72 meses após o preparo do solo, não foi observada diferença significativa entre os métodos de preparo do solo para as variáveis de solo estudadas e para o crescimento de Eucalyptus grandis. Todavia, foi observada diferença significativa para a sobrevivência das árvores, com menor porcentagem para o coveamento manual, o qual exibiu uma redução expressiva do número de árvores quando comparado com os outros métodos de preparo do solo estudados. Dessa forma, o coveamento manual pode não ser recomendado para o plantio de Eucalyptus grandis nas condições edafoclimáticas do presente estudo.

A desagregação do solo observada logo após seu preparo por Prevello (2008) deixou de existir aos 72 meses após o preparo do solo, e a condição física do solo apresentou-se semelhante ou melhor que a encontrada antes da implantação experimento. A melhoria das propriedades físicas do solo foi evidenciada pelo aumento do conteúdo de matéria orgânica na superfície do solo, promovendo o aumento da porosidade, a redução da densidade do solo e, possivelmente, a melhoria da aeração e infiltração da água no solo. A influência das árvores, por meio do aporte de serapilheira e do sistema radicular, teve destacada importância para a melhora da condição física do solo.

\section{REFERÊNCIAS BIBLIOGRÁFICAS}

AMARO FILHO, J.; ASSIS JR., R. N.; MOTA, J. C. A. Física do Solo: Conceitos e Aplicações. Fortaleza: Imprensa Universitária, 2008. 290 p.

BAPTISTA, J.; LEVIEN, R. Métodos de preparo de solo e sua influência na erosão hídrica e no acúmulo de biomassa da parte aérea de Eucalyptus saligna em um Cambissolo Háplico da depressão central do Rio Grande do Sul. Revista Árvore, Viçosa, v. 34, n. 4, p. 567-575, 2010. 
BERTONI, J.; LOMBARDI NETO, F. Conservação do solo. 6.ed. São Paulo: Ícone, 2008. 355 p.

BRADY, N. C.; WEIL, R. R. Elementos da natureza e propriedades dos solos. 3.ed. Porto Alegre: Bookman, 2013. $685 \mathrm{p}$.

BRAIDA, J. A.; BAYER, C.; ALBUQUERQUE, J. A.; REICHERT, J. M. Matéria orgânica e seu efeito na física do solo. In: KLAUBERG FILHO, O.; MAFRA, A. L.; GATIBONI, L. C. (Org.). Tópicos em ciência do solo. Viçosa: SBCS, 2011. v. VII, p.222-227.

BRANDÃO, E. D.; SILVA, I. F. Formação e estabilização de agregados pelo sistema radicular de braquiária em um Nitossolo Vermelho. Ciência Rural, Santa Maria, v. 42, n. 7, p. 1193-1199, 2012.

CASTRO FILHO, C.; MUZILLI, O.; PODANOSCHI, A. L. Estabilidade dos agregados e sua relação com o teor de carbono orgânico num Latossolo Roxo distrófico, em função de sistemas de plantio, rotações de culturas e métodos de preparo das amostras. Revista Brasileira de Ciência do Solo, Viçosa, v. 22, n. 3, p. 527-538, 1998.

CQFS-RS/SC - COMISSÃO DE QUÍMICA E FERTILIDADE DO SOLO - RS/SC. Manual de adubação e de calagem para os Estados do Rio Grande do Sul e de Santa Catarina. 10.ed. Porto Alegre: SBCS, 2004.400 p.

EMBRAPA - EMPRESA BRASILEIRA DE PESQUISA AGROPECUÁRIA. Manual de métodos de análise de solo. Rio de Janeiro: EMBRAPA-CNPS, 1997. 212 p.

EMBRAPA - EMPRESA BRASILEIRA DE PESQUISA AGROPECUÁRIA. Sistema Brasileiro de Classificação de Solos. 2.ed. Brasília: Embrapa, 2013. 306 p.

FERREIRA, M. M. Caracterização Física do Solo. In: VAN LIER, Q. J. (Ed.). Física do solo. Viçosa: SBCS, 2010. Cap. 1. p. 1-27.

FINGER, C. A. G.; SCHUMACHER, M. V.; SCHNEIDER, P. R.; HOPPE, J. M. Influência da camada de impedimento no solo sobre o crescimento de Eucalyptus grandis (Hill) ex Maiden. Ciência Florestal, Santa Maria, v. 6, n. 1, p. 137-145, 1996.

GATTO, A.; BARROS, N. F.; NOVAIS, R. F.; COSTA, L. M.; NEVES, J. C. L. Efeito do método de preparo do solo, em área de reforma, nas suas características, na composição mineral e na produtividade de plantações de Eucalyptus grandis. Revista Árvore, Viçosa, v. 27, n. 5, p. 635-646, 2003.

GONÇALVES, J. L. M. Estabelecimento de plantações de eucaliptos usando o sistema de cultivo mínimo do solo. In: JORNADAS FORESTALES DE ENTRE RIOS, 10., 1995, Cocordia. Actas... Concordia : INTA, 1995. p. II.1-II.15.

GONÇALVES, J. L. M.; STAPE, J. L. (Ed.) Conservação e cultivo de solos para plantações florestais. 1.ed. Piracicaba: IPEF, 2002. 498 p.

GONZÁLEZ, P.; PÉREZ BIDEGAIN, M.; GUTIÉRREZ, L.; MARTINEZ, L.; GARCÍA PRÉCHAC, F. Evaluation of different tillage intensities on Eucalyptus grandis on a typic Hapludult of Uruguay. Agrociencia Uruguay, v. 16, n. 3, p. 302-305, 2012.

KEMPER, W. D.; CHEPIL, W. S. Size distribution of aggregates. In: BLACK, C. A.; EVANS, D. D.; WHITE, J. L. (Eds.). Methods of soil analysis. Madison: American Society of Agronomy, 1965. p. 499-509.

KIEHL, E. J. Manual de edafologia: relações solo-planta. São Paulo: Ed. Agronômica Ceres, 1979. 264 p. 
Rocha et al. - Propriedades físicas do solo e crescimento de

eucalipto implantado em diferentes métodos de preparo do solo

LAURINDO, M. C. O.; NÓBREGA, L. H. P.; PEREIRA, J. O.; MELO, D.; LAURINDO, É. L. Atributos físicos do solo e teor de carbono orgânico em sistemas de plantio direto e cultivo mínimo. Engenharia na agricultura, Viçosa, n. 17, p. 367-374, 2009.

LETEY, J. Relationship between soil physical properties and crop production. Advances in Soil Science, v. 1, p. 277-294, 1985. MALUF, J. R. T. Nova classificação climática do Estado do Rio Grande do Sul. Revista Brasileira de Agrometeorologia, Santa Maria, v. 8, n. 1, p. 141-150, 2000.

MENDHAM, D. S. Soil particulate organic matter effects on nitrogen availability after afforestation with Eucalyptus globulus. Soil Biology and Biochemistry, Oxford, v. 36, n. 7, p.1067-1074, 2004.

MISHRA, A.; SHARMA, S. D.; KHAN, G. H. Improvement in physical and chemical properties of sodic soil by 3, 6 and 9 years old plantation of Eucalyptus tereticornis Biorejuvenation of sodic soil. Forest Ecology and Management, Amsterdam, v. 184, n. 1-3, p. 115-124, 2003.

MONTERO, L. L. Carbono em solos do serrado: efeitos do uso florestal. 2008. 130 p. Tese (Doutorado em Ciências) - Universidade de São Paulo, São Paulo, 2008.

MORENO, J. A. Clima do Rio Grande do Sul. Porto Alegre: Secretaria da agricultura, 1961. 42 p.

NEVES, E. J. M.; MARTINS, E. G.; REISSMANN, C. B. Deposição de serapilheira e de nutrientes de duas espécies da Amazônia. Boletim de Pesquisa Florestal, Colombo, n. 43, p. 47-60, 2001.

PAES, F. A. S. V.; LIMA, A. M. N.; HAKAMADA, R. E.; BARROS, N. F. Impacto do manejo dos resíduos da colheita, do preparo do solo e da adubação na produtividade de eucalipto. Revista Brasileira de Ciência do Solo, Viçosa, v. 37, n. 4, p. 1081-1090, 2013.

PICCOLO, A.; PIETRAMELLARA, G.; MBAGWU, J. S. C. Use of humic substances as soil conditioners to increase aggregate stability. Geoderma, v. 75, n. 3, p. 267-277, 1997.

PREVEDELLO, J. Preparo do solo e crescimento inicial de Eucalyptus grandis Hill ex Maiden. em Argissolo. 2008. 81 p. Dissertação (Mestrado em Engenharia Florestal) - Universidade Federal de Santa Maria, Santa Maria, 2008.

REICHERT, J. M.; KAISER, D. R.; REINERT, D. J.; RIQUELME, U. F. B. Variação temporal de propriedades físicas do solo e crescimento radicular de feijoeiro em quatro sistemas de manejo. Pesquisa Agropecuária Brasileira, Brasília, v. 44, n. 3, p. 310-319, 2009.

REICHERT, J. M.; REINERT, D. J.; BRAIDA, J. A. Qualidade dos Solos e sustentabilidade de sistemas agrícolas. Revista Ciência \& Ambiente, Santa Maria, v. 27, n. 1, p. 29-48, 2003.

ROSA, D. P.; REICHERT, J. M.; SATTLER, A.; REINERT, D. J.; MENTGES, M. I.; VIEIRA, D. A. Relação entre solo e haste sulcadora de semeadora em Latossolo escarificado em diferentes épocas. Pesquisa Agropecuária Brasileira, Brasília, v. 43, n. 3, p. 395-400, mar. 2008.

ROZANE, D. E.; CENTURION, J. F.; ROMUALDO, L. M.; TANIGUCHI, C. A. K.; TRABUCO, M.; ALVES, A. U. Estoque de carbono e estabilidade de agregados de um Latossolo Vermelho distrófico, sob diferentes manejos. Bioscience Journal, Uberlândia, v. 26, n. 1, p. 24-32, jan./fev. 2010.

SILVA, D. A.; SOUZA, L. C. F.; VITORINO, A. C. T.; GONÇALVES, M. C. Aporte de fitomassa pelas sucessões de culturas e sua influência em atributos físicos do solo no sistema plantio direto. Bragantia, Campinas, v. 70, n. 1, p.147-156, 2011. 
SILVA, E. V.; GONÇALVES, J. L. M.; COELHO, S. R. F.; MOREIRA, R. M.; MELLO, S. L. M.; BOUILLET, J. P.; JOURDAN, C.; LACLAU, J. P. Dynamics of fine root distribution after establishment of monospecific and mixed-species plantations of Eucalyptus grandis and Acacia mangium. Plant Soil, v. 325, n. 1, p. 305-318, 2009.

SILVA, I. F.; MIELNICZUK, J. Sistemas de cultivo e características do solo afetando a estabilidade de agregados. Revista Brasileira de Ciência do Solo, Viçosa, v. 22, n. 2, p. 311-317, 1998.

TEDESCO, M. J.; GIANELLO, C.; BISSANI, C. A.; BOHNEN, H.; WOLKWEISS, S. J. Análise de solo, plantas e outros materiais. 2.ed. Porto Alegre: Ed. da UFRGS, 1995. 174 p. (Boletim Técnico, 5).

THEODORO, V. C. A.; ALVARENGA, M. I. N.; GUIMARÃES, R. J.; MOURÃO JR., M. Propriedades físicas de um Latossolo Vermelho-Escuro sob mata nativa e sistemas de produção de café orgânico, em conversão e convencional. In: SIMPÓSIO DE PESQUISA DOS CAFÉS NO BRASIL, 2., 2001, Vitória. Anais... Vitória: EMBRAPA, 2001. 1 CD-ROM.

TISDALL, J. M.; OADES, J. M. Organic matter and water-stable aggregates in soil. Journal of Soil Science, v. 33, n. 2, p. 141-163, 1982.

TORMENA, C. A. Densidade, porosidade e resistência à penetração em Latossolo cultivado sob diferentes sistemas de preparo do solo. Scientia Agricola, Piracicaba, v. 59, n. 4, p. 795-801, 2002.

VEZANI, F. M.; MIELNICZUK, J. Uma visão sobre qualidade do solo. Revista Brasileira de Ciência do Solo, Viçosa, v. 33, n. 4, p. 743-755, 2009.

XU, X.; NIEBER, J. L.; GUPTA, S. C. Compaction effect on the gas diffusion coefficient in soils. Soil Science Society of America Journal, Madison, v. 56, n. 6, p. 1743-1750, 1992.

YODER, R. E. A direct method of aggregate analysis of soils and a study of the physical nature of erosion losses. Journal American Society of Agronomy, v. 28. p. 337-351, 1936.

ZINN, Y. L.; LAL, R.; RESCK, D. V. S. Eucalypt plantation effects on organic carbon and aggregation of three different-textured soils in Brazil. Soil Research, Collingwood, v. 49, n. 7, p. 614-624, 2011.

ZONTA E.; BRASIL, F. C.; GOI, S. R.; ROSA, M. M. T. O sistema radicular e suas interações com o ambiente edáfico. In: FERNANDES, M. S. (Ed.). Nutrição mineral de plantas. Viçosa: SBCS, 2006. p. 7-52.

Recebido em 02/09/2014

Aceito para publicação em 25/05/2015

Sci. For., Piracicaba, v. 43, n. 108, p. 965-977, dez. 2015 DOI: dx.doi.org/10.18671/scifor.v43n108.20 
